University of Wollongong

Research Online

Faculty of Engineering and Information

Faculty of Engineering and Information

Sciences - Papers: Part B

Sciences

2017

A new closed-form formula for pricing European options under a skew Brownian motion

\author{
Song-Ping Zhu \\ University of Wollongong, spz@uow.edu.au \\ Xin-Jiang He \\ University of Wollongong, xh016@uowmail.edu.au
}

Follow this and additional works at: https://ro.uow.edu.au/eispapers1

Part of the Engineering Commons, and the Science and Technology Studies Commons

Research Online is the open access institutional repository for the University of Wollongong. For further information contact the UOW Library: research-pubs@uow.edu.au 


\title{
A new closed-form formula for pricing European options under a skew Brownian motion
}

\begin{abstract}
In this paper, we present a new pricing formula based on a modified Black-Scholes (B-S) model with the standard Brownian motion being replaced by a particular process constructed with a special type of skew Brownian motions. Although Corns and Satchell [2007. "Skew Brownian Motion and Pricing European Options." The European Journal of Finance 13 (6): 523-544] have worked on this model, the results they obtained are incorrect. In this paper, not only do we identify precisely where the errors in Although Corns and Satchell [2007. "Skew Brownian Motion and Pricing European Options". The European Journal of Finance 13 (6): 523-544] are, we also present a new closed-form pricing formula based on a newly proposed equivalent martingale measure, called 'endogenous risk neutral measure', by which only endogenous risks should and can be fully hedged. The newly derived option pricing formula takes the B-S formula as a special case and it does not induce any significant additional burden in terms of numerically computing option values, compared with the effort involved in computing the B-S formula.

\section{Disciplines}

Engineering | Science and Technology Studies

\section{Publication Details}

Zhu, S. \& He, X. (2017). A new closed-form formula for pricing European options under a skew Brownian motion. The European Journal of Finance, Online First 1-13.
\end{abstract}




\title{
A new closed-form formula for pricing European options under a skew Brownian motion
}

\author{
Song-Ping $\mathrm{ZHU}^{*} \quad$ Xin-Jiang $\mathrm{HE}^{\dagger}$
}

\begin{abstract}
In this paper, we present a new pricing formula based on a modified Black-Scholes model with the standard Brownian motion being replaced by a particular process constructed with a special type of skew Brownian motions. Although Corns \& Satchell [4] have worked on this model, the results they obtained are incorrect. In this paper, not only do we identify precisely where the errors in [4] are, we also present a new closed-form pricing formula based on a newly proposed equivalent martingale measure, called "endogenous risk neutral measure", by which only endogenous risks should and can be fully hedged. The newly derived option pricing formula takes the Black-Scholes formula as a special case and it does not induce any significant additional burden in terms of numerically computing option values, compared with the effort involved in computing the Black-Scholes formula.
\end{abstract}

Keywords. Option pricing, Skew Brownian motion, Non-normal distribution.

${ }^{*}$ Corresponding author. School of Mathematics and Applied Statistics, University of Wollongong NSW 2522, Australia. Email: spz@uow.edu.au.

${ }^{\dagger}$ School of Mathematics and Applied Statistics, University of Wollongong NSW 2522, Australia. 


\section{Introduction}

Although a great breakthrough took place in 1973 when Black \& Scholes [2] assumed that the log-returns of the underlying price follows a normal distribution and obtained a closed-form option pricing formula, there are some drawbacks in the B-S (Black-Scholes) model because of some unrealistic assumptions made to achieve analytical simplicity and tractability. A typical example is that the normality of the log-return model can not capture the features like skewness [14] and time-dependence [9] that appear in real market data.

In the literature, many attempts have been made to modify the distributional assumptions of the B-S model. Among them, Lévy processes are really popular since they have independent and stationary increments, a similar property of which can be found in a Brownian motion. In particular, jump-diffusion models, as one kind of the most popular Lévy processes, have received a lot of attention. For example, Merton [12] proposed a model whose jumps in the log-returns have a Gaussian distribution, while Kou [10] adopted another one with the jump size having an asymmetric double exponential distribution. Another sub-category is infinite activity lévy process where the Brownian component is omitted. Madan [11] introduced the Variance-Gamma model which is a time-changed Brownian motion. Others in this category include Normal Inverse Gaussian process used by Rydberg [15] and CGMY model proposed by Carr et al. [3].

A stochastic process considered here to model the dynamics of the underlying price is related to skew Brownian motions. Actually, skew Brownian motions were firstly introduced by Itô \& McKean [8]. They are characterized by a skew parameter $\alpha \in[0,1]$, and their excursion from zero has the probability $\alpha$ to be positive and the probability $1-\alpha$ to be negative. Recently, several authors have adopted skew Brownian motions and skewnormal distributions in financial modeling. For example, it has been pointed out by Eling

et al. [5] that the Azzalini skew-normal distribution [1], which is the probability density of 
the adopted skew Brownian motion in this paper, has a number of advantages over common measures of skewness. Moreover, Nilsen \& Sayit [13] even went further and argued that skew Brownian motions were able to describe the bounces and sinks of financial firms in distress. Furthermore, Corns \& Satchell [4] and Gairat \& Shcherbakov [7] used them in derivative pricing. In particular, Gairat \& Shcherbakov built a relationship between discontinuous local volatility models and skew Brownian motions for option pricing, while Corns \& Satchell [4] were the first to derive a pricing formula for European options in terms of adopting skew Brownian motions directly in asset modeling. Unfortunately, the results Corns \& Satchell [4] provided are completely wrong, which will be proven later.

In this paper, we choose a typical type of skew Brownian motions, constructed by a linear combination of a standard Brownian motion and a standard reflected Brownian motion, whose density function is a skew-normal distribution. By adopting the particular kind of skew Brownian motions in option pricing, the non-normality property is introduced in the distribution of log-returns. We will firstly show the results in [4] are incorrect and then use the martingale approach to derive a new formula for European call option prices. In the process of developing this new pricing formula, another key contribution of this paper emerged, as a result of an assumption we made to split the risks associated with the underlying into two parts. The first is an endogenous part that measures the risks within the financial system which is somewhat controllable as they are mainly due to management and people's interaction with each other, while the second is an exogenous part that is referred to as the risks caused by external factors, over which we have no control. If such a split is possible in a market environment that the underlying is in, then a risk neutral martingale measure called "endogenous risk neutral measure" can be adopted. It is based on this new concept that our pricing formula was derived.

The rest of the paper is organized as follows. In Section 2, we will point out and confirm two errors contained in [4], which leads to a totally wrong pricing formula. In Section 3, the real challenge is to find a new equivalent martingale measure, based on 
which we are able to derive a closed-form analytical solution for European option prices under the adopted model. In Section 4, numerical examples and some useful discussions are presented. Concluding remarks are given in the last section.

\section{Incorrectness of a key pricing formula}

As pointed out in the previous section, Corns \& Satchell [4] option pricing formula was a key pricing formula in closed form with a skew Brownian motion being adopted to model the dynamics of the underlying price. Unfortunately, there are two key errors in their formula. To demonstrate that the errors are not simple typos and the correction requires

a careful choice of a new risk-neutral measure, two lemmas need to be introduced first in this section.

Lemma 2.1 Let $X$ be a random variable with its probability density function

$$
f_{X}(x)=\frac{2}{\sigma x} \phi\left(\frac{\ln x-u}{\sigma}\right) \Phi\left(\lambda \frac{\ln x-u}{\sigma}\right),
$$

where $\phi$ and $\Phi$ represent a standard normal density and distribution function, respectively. Then if $Y=\ln X$, the probability density function of $Y$ is

$$
f_{Y}(y)=\frac{2}{\sigma} \phi\left(\frac{y-u}{\sigma}\right) \Phi\left(\lambda \frac{y-u}{\sigma}\right) .
$$

Proof.

$$
F_{Y}(y)=P(Y \leq y)=P(\ln X \leq y)=P\left(X \leq e^{y}\right)=F_{X}\left(e^{y}\right),
$$

from which we can derive

$$
f_{Y}(y)=e^{y} f_{X}\left(e^{y}\right)=\frac{2}{\sigma} \phi\left(\frac{y-u}{\sigma}\right) \Phi\left(\lambda \frac{y-u}{\sigma}\right) .
$$


Lemma 2.2 Let $Z$ be a random variable with its density function

$$
f_{Z}(z)=\frac{2}{\sigma} \phi\left(\frac{z-u}{\sigma}\right) \Phi\left(\lambda \frac{z-u}{\sigma}\right)
$$

where $\phi$ and $\Phi$ represent a standard normal density and distribution function, respectively. Then, $E(Z)=\sigma \delta \sqrt{\frac{2}{\pi}}+u$.

Proof. The moment generating function of $Z$ can be calculated as

$$
\begin{aligned}
M_{Z}(\theta) & =E\left(e^{\theta Z}\right)=\int_{-\infty}^{\infty} \frac{2}{\sigma} e^{\theta z} \phi\left(\frac{z-u}{\sigma}\right) \Phi\left(\lambda \frac{z-u}{\sigma}\right) d z \\
& =\frac{2}{\sigma \sqrt{2 \pi}} e^{u \theta+0.5 \theta^{2} \sigma^{2}} \int_{-\infty}^{\infty} e^{-\frac{y^{2}}{2 \sigma^{2}}} \Phi\left(\lambda \frac{y}{\sigma}+\lambda \theta \sigma\right) d y=2 e^{u \theta+0.5 \theta^{2} \sigma^{2}} \Phi\left(\frac{\lambda \theta \sigma}{\sqrt{1+\lambda^{2}}}\right)
\end{aligned}
$$

The last step is according to Lemma 2 in [1]. Moreover, if we set $\delta=\frac{\lambda}{\sqrt{1+\lambda^{2}}}$, we then have

$$
\frac{\partial\left(E\left(e^{\theta Z}\right)\right)}{\partial \theta}=\frac{\partial\left(2 e^{u \theta+0.5 \theta^{2} \sigma^{2}} \Phi(\delta \theta \sigma)\right)}{\partial \theta}
$$

which can be simplified to

$$
E\left(Z e^{\theta Z}\right)=2 e^{u \theta+0.5 \theta^{2} \sigma^{2}}\left(u+\theta \sigma^{2}\right) \Phi(\delta \theta \sigma)+2 e^{u \theta+0.5 \theta^{2} \sigma^{2}} \phi(\delta \theta \sigma) \delta \sigma .
$$

Setting $\theta=0$ in Equation (2.3) leads to the desired result.

Now, let $S_{t}$ denote the underlying asset price at time $t$ and $f\left(S_{T} \mid S_{t}\right)$ be the probability density function of $S_{T}$ conditional upon $S_{t}$ for $t<T$ as defined in [4]. Then, it needs to be pointed out that the expression of $f\left(S_{T} \mid S_{t}\right)$, which is used for option pricing in [4], was wrongly derived from the expression of $S_{t}$.

We shall prove their error by contradiction. On one hand, according to [4],

$$
f\left(S_{T} \mid S_{t}\right)=\frac{2}{\sigma S_{T} \sqrt{T-t}} \phi(y) \Phi(\lambda y)
$$


where $y=\frac{\ln S_{T}-\ln S_{t}-\left(r-0.5 \sigma^{2}\right)(T-t)+\ln [2 \Phi(\delta \sigma \sqrt{T-t})]}{\sigma \sqrt{T-t}}$, which implies $\ln S_{T}=$ $\sigma \sqrt{T-t} y+\ln S_{t}+\left(r-0.5 \sigma^{2}\right)(T-t)-\ln [2 \Phi(\delta \sigma \sqrt{T-t})]$. Thus, with Lemma 2.1 we can further obtain

$$
f\left(\ln S_{T} \mid S_{t}\right)=\frac{2}{\sigma \sqrt{T-t}} \phi(y) \Phi(\lambda y)
$$

from which we can calculate

$$
\begin{aligned}
E\left(\ln S_{T} \mid S_{t}\right) & =\int_{-\infty}^{\infty} \ln S_{T} f\left(\ln S_{T} \mid S_{t}\right) d\left(\ln S_{T}\right) \\
& =\ln S_{t}+\left(r-0.5 \sigma^{2}\right)(T-t)-\ln [2 \Phi(\delta \sigma \sqrt{T-t})]+\sigma \sqrt{T-t} \int_{-\infty}^{\infty} 2 y \phi(y) \Phi(\lambda y) d y \\
& =\ln S_{t}+\left(r-0.5 \sigma^{2}\right)(T-t)-\ln [2 \Phi(\delta \sigma \sqrt{T-t})]+\delta \sigma \sqrt{\frac{2(T-t)}{\pi}}
\end{aligned}
$$

The last step results from Lemma 2.2.

On the other hand, Equation (12) in [4], i.e.,

$$
S_{t}=S_{0} e^{\left(r-0.5 \sigma^{2}\right) t-\ln [2 \Phi(\delta \sigma \sqrt{t})]+\sigma \bar{X}_{t}},
$$

with $\bar{X}_{t}$ being a $\mathbb{Q}$ skew Brownian motion, can lead to

$$
\begin{aligned}
\ln S_{t} & =\ln S_{0}+\left(r-0.5 \sigma^{2}\right) t-\ln [2 \Phi(\delta \sigma \sqrt{t})]+\sigma \bar{X}_{t}, \\
\ln S_{T} & =\ln S_{0}+\left(r-0.5 \sigma^{2}\right) T-\ln [2 \Phi(\delta \sigma \sqrt{T})]+\sigma \bar{X}_{T} .
\end{aligned}
$$

The combination of these two equations further yields

$$
\ln S_{T}-\ln S_{t}=\left(r-0.5 \sigma^{2}\right)(T-t)-\{\ln [2 \Phi(\delta \sigma \sqrt{T})]-\ln [2 \Phi(\delta \sigma \sqrt{t})]\}+\sigma\left(\bar{X}_{T}-\bar{X}_{t}\right),
$$


which leads to another expression of $E\left(\ln S_{T} \mid S_{t}\right)$ as

$E\left(\ln S_{T} \mid S_{t}\right)=\ln S_{t}+\left(r-0.5 \sigma^{2}\right)(T-t)-\{\ln [2 \Phi(\delta \sigma \sqrt{T})]-\ln [2 \Phi(\delta \sigma \sqrt{t})]\}+E\left[\sigma\left(\bar{X}_{T}-\bar{X}_{t}\right) \mid S_{t}\right]$.

Subtracting Equation (2.7) from Equation (2.5) on both sides respectively and taking expectation from the both sides of the resulted equation lead to

$$
E\left[\sigma\left(\bar{X}_{T}-\bar{X}_{t}\right)\right]=\ln [2 \Phi(\delta \sigma \sqrt{T})]-\ln [2 \Phi(\delta \sigma \sqrt{t})]-\ln [2 \Phi(\delta \sigma \sqrt{T-t})]+\sigma \delta \sqrt{\frac{2}{\pi}}(\sqrt{T-t}) .
$$

Given Lemma 2.2, we can also obtain $E\left[\sigma\left(\bar{X}_{T}-\bar{X}_{t}\right)\right]=\sigma \delta \sqrt{\frac{2}{\pi}}(\sqrt{T}-\sqrt{t})$. Thereby, we finally arrive at

$$
\sigma \delta \sqrt{\frac{2}{\pi}}(\sqrt{T}-\sqrt{t}-\sqrt{T-t})=\ln [2 \Phi(\delta \sigma \sqrt{T})]-\ln [2 \Phi(\delta \sigma \sqrt{t})]-\ln [2 \Phi(\delta \sigma \sqrt{T-t})]
$$

which should hold if (2.4) was correct.

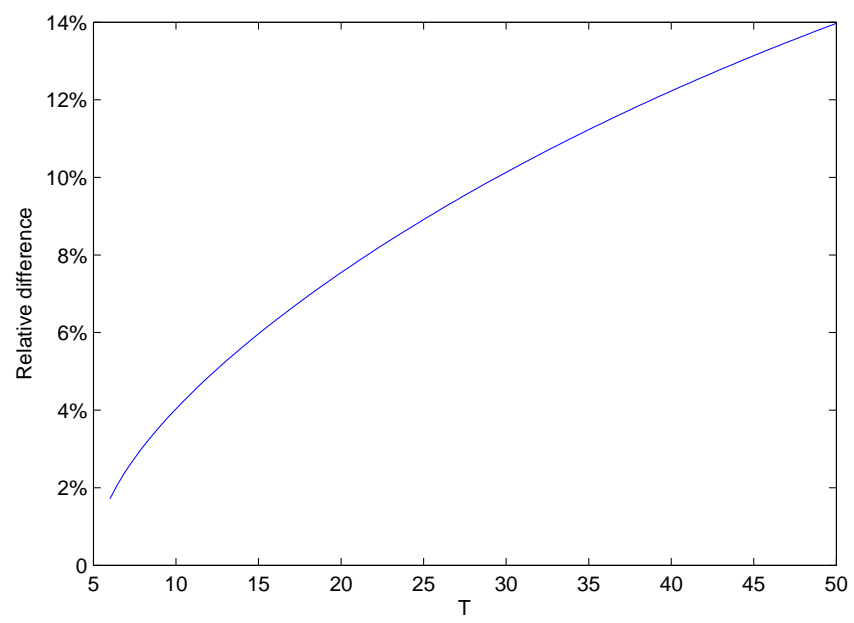

Figure 1: Differences among the two sides of Equation (2.8).

To prove an equation wrong, all we need to do is to show that it does not hold for a single set of parameters. Clearly, Equation (2.8) does not hold as we can easily find not only one, but a set of continuous points in the parameter space such that the difference 
between the two sides of Equation (2.8) is far from zero. In particular, plotted in Fig. 1 are percentage differences between the two sides of Equation (2.8) with $\delta=0.5, \sigma=\sqrt{0.4}$ and $t=5$, when $T-t$ is varied in the range $[1,45]$. Indeed, the maximum difference for this set of parameters reaches 14\%, which implies that (2.8) does not hold and thus completes our proof through contradiction. The incorrectness of (2.4) naturally leads us to draw a conclusion that the pricing formula (14) in [4] is incorrect.

We could not exactly identify where the mistake was made until we had realized that Corns \& Satchell [4] had taken the extension of $f\left(\ln S_{t} \mid S_{0}\right)$ to $f\left(\ln S_{T} \mid S_{t}\right)$ for granted, which is not true since reflected Brownian motions do not have a stationary increment. As we dug further, we eventually found another error that they had regarded $E^{Q}\left[e^{-r T} S_{T} \mid F_{0}\right]=S_{0}$ as the condition for the discounted asset price to be a martingale. However, this condition is actually only a necessary but not sufficient condition for $e^{-r t} S_{t}$ to be a martingale, which again shows that the obtained option price, based on a wrongly chosen condition, should not be a fair price. In the following section, we will identify a risk-neutral measure with respect to the chosen geometric skew Brownian motions, and then present a correct option pricing formula with the full martingale condition imposed.

\section{New Formula}

In this section, the dynamics for the underlying price and a new martingale measure are introduced first. Then, a martingale method is used to derive a pricing formula for European call options.

\subsection{A new equivalent martingale measure}

Let $(\Omega, F, P)$ define a probability space where $F$ is a $\sigma$-algebra on $\Omega$ and $P$ is a probability measure on $(\Omega, F)$. We now first assume that the underlying price process under the 
physical measure follows the same process in [4], i.e.,

$$
S_{T}=S_{t} e^{\mu(T-t)+\sigma\left(X_{T}-X_{t}\right)},
$$

where $t$ is the current time, and $X_{s}$ is a skew Brownian motion defined as

$$
X_{s}=\sqrt{1-\delta^{2}} W_{1, s}+\delta\left|W_{2, s}\right|, \quad \delta \in(-1,1)
$$

with $W_{1}$ and $W_{2}$ being two independent standard Brownian motions. The probability density function of $X_{s}$ is

$$
f_{X_{s}}(x)=\frac{2}{\sqrt{s}} \phi\left(\frac{x}{\sqrt{s}}\right) \Phi\left(\lambda \frac{x}{\sqrt{s}}\right)
$$

As we mentioned in the last section that the martingale measure presented in [4] is not a correct one, which is due to the fact that the discounted underlying is not a local martingale under this measure, we need to find a new martingale measure $\mathbb{Q}$, under which $e^{-r s} S_{s}$ is a martingale. If we further let

$$
W_{s}=\sqrt{1-\delta^{2}} W_{1, s}, \quad R_{s}=\delta\left|W_{2, s}\right|
$$

it is not difficult to obtain the following conditional expectation

$$
E^{P}\left[e^{-r T} S_{T} \mid F_{t}\right]=e^{-r T} S_{t} e^{\mu(T-t)-\sigma W_{t}-\sigma X_{t}} E^{P}\left[e^{\sigma W_{T}+\sigma R_{T}} \mid F_{t}\right]
$$

Also, vectors $\left(\bar{W}_{T}, \bar{W}_{t}\right)$ and $\left(\bar{R}_{T}, \bar{R}_{t}\right)$ are independent of each other $(\forall t<T)$ since $W_{T}$ and $R_{T}$ are independent, and thus we can obtain

$$
\begin{aligned}
f_{\sigma W_{T}, \sigma R_{T} \mid \sigma W_{t}, \sigma R_{t}} & =\frac{f_{\sigma W_{T}, \sigma R_{T}, \sigma W_{t}, \sigma R_{t}}}{f_{\sigma W_{t}, \sigma R_{t}}}=\frac{f_{\sigma W_{T}, \sigma W_{t}} \cdot f_{\sigma R_{T}, \sigma R_{t}}}{f_{\sigma W_{t}} \cdot f_{\sigma R_{t}}} \\
& =f_{\sigma W_{T} \mid \sigma W_{t}} \cdot f_{\sigma R_{T} \mid \sigma R_{t}} .
\end{aligned}
$$


Furthermore, we can easily show that

$$
\begin{gathered}
f_{\sigma W_{T} \mid \sigma W_{t}}\left(x_{1} \mid y\right)=\frac{1}{\sigma \sqrt{2 \pi(T-t)\left(1-\delta^{2}\right)}} e^{-\frac{\left(x_{1}-y\right)^{2}}{2(T-t)\left(1-\delta^{2}\right) \sigma^{2}}}, \\
f_{\sigma R_{T} \mid \sigma R_{t}}\left(x_{2} \mid z\right)=\frac{1}{\delta \sigma \sqrt{2 \pi(T-t)}}\left(e^{-\frac{\left(x_{2}-z\right)^{2}}{2(T-t) \delta^{2} \sigma^{2}}}+e^{-\frac{\left(x_{2}+z\right)^{2}}{2(T-t) \delta^{2} \sigma^{2}}}\right) .
\end{gathered}
$$

Thus, the conditional expectation, $E^{P}\left[e^{\sigma W_{T}+\sigma R_{T}} \mid F_{t}\right]$, can be worked out as

$$
\begin{aligned}
E^{P}\left[e^{\sigma W_{T}+\sigma R_{T}} \mid F_{t}\right] & =\int_{0}^{+\infty} \int_{-\infty}^{+\infty} e^{x_{1}+x_{2}} f_{\sigma W_{T}, \sigma R_{T} \mid \sigma W_{t}, \sigma R_{t}}\left(x_{1}, x_{2} \mid y, z\right) d x_{1} d x_{2} \\
& =e^{y+z+0.5 \sigma^{2}(T-t)} e^{l(z)}
\end{aligned}
$$

with $y=W_{t}, z=R_{t}$, and $l(z)=\ln \left(\Phi\left[\frac{z+(T-t) \sigma^{2} \delta^{2}}{\sqrt{T-t} \sigma \delta}\right]+e^{-2 z} \Phi\left[\frac{-z+(T-t) \sigma^{2} \delta^{2}}{\sqrt{T-t} \sigma \delta}\right]\right)$. Consequently, the substitution of Equation (3.7) into (3.3) finally yields

$$
E^{P}\left[e^{-r T} S_{T} \mid F_{t}\right]=e^{-r t} S_{t} e^{\left(\mu-r+0.5 \sigma^{2}\right)(T-t)+l(z)} .
$$

It should be pointed out that it is very difficult to find an equivalent martingale measure for the underlying price process (3.1) due to the complicated expression of $l(z)$. Therefore, we introduce an "adjusted" drift term so that the underlying price process under the physical measure follows

$$
S_{T}=S_{t} e^{\mu(T-t)-l(z)+\sigma\left(X_{T}-X_{t}\right)} .
$$

In this case, if we make the measure transform of

$$
\begin{aligned}
& \bar{W}_{1, t}=W_{1, t}+\frac{\left[\mu-r+0.5 \sigma^{2}\right] t}{\sigma \sqrt{1-\delta^{2}}}, \\
& \bar{W}_{2, t}=W_{2, t}
\end{aligned}
$$

where $\bar{W}_{1}$ and $\bar{W}_{2}$ are independent Brownian motions under the equivalent martingale 
measure $\mathbb{Q}$, and define $\bar{X}_{T}=\sqrt{1-\delta^{2}} \bar{W}_{1, T}+\delta\left|\bar{W}_{2, T}\right|$, we can certainly obtain

$$
S_{T}=S_{t} e^{\left(r-0.5 \sigma^{2}\right)(T-t)-l(z)+\sigma\left(\bar{X}_{T}-\bar{X}_{t}\right)}
$$

with $E^{Q}\left[e^{-r T} S_{T} \mid F_{t}\right]=e^{-r t} S_{t}$. This demonstrates that $\mathbb{Q}$ is an equivalent martingale measure. Clearly, our model is essentially different from that used in [7] as the adopted skew Brownian motion in this paper possesses a time-dependent drift term while the one they chose has a two-valued drift term, which is not continuous.

It should also be noted that our choice for this particular martingale measure is motivated by the so-called "minimal martingale measure" [16] in the sense that we assume only risks associated with the Brownian motion $W_{1}$ need to be hedged. To be more specific, the stochastic process constructed with $W_{1}$ represents endogenous risks of the underlying while the stochastic process constructed with $W_{2}$ models all exogenous risks. In this sense, we keep $W_{2}$ unchanged in performing the measure transform since we have no control over exogenous risks associated with $W_{2}$, and we only focus on hedging endogenous risks associated with $W_{1}$ through a shift of the mean to $W_{1}$ performed in Equation (3.9). For this reason, we have named this particular martingale measure "endogenous risk neutral measure" to suggest that only risks associated with the endogenous process can and should be fully hedged.

In fact, this concept of splitting total risks into two parts, namely endogenous risks and exogenous risks, and then pricing a financial derivative with an "endogenous risk neutral measure" can be extended to other option pricing models. Of course, the key to the success hinges on the situation where we are able to find an equivalent martingale measure by only shifting one stochastic source. On the other hand, like some other approaches proposed in the literature to price financial derivatives in incomplete markets, such as [6, 16], our proposed "endogenous risk neutral measure" approach adds a new "flavor" to the literature; whether or not it is superior to other approaches are yet to be tested by empirical studies. 


\subsection{A new closed-form option pricing formula}

Now we are ready to derive the option pricing formula under $\mathbb{Q}$.

Proposition 3.1 Let the underlying asset price $S_{t}$ follow the particular process (3.10) under the martingale measure $\mathbb{Q}$ that was derived in the last subsection, then the European call option pricing formula can be written as

$$
C(S, t)=S_{t} M_{1}\left(b_{1}\right)-K e^{-r(T-t)} M_{2}\left(b_{2}\right),
$$

where $M_{1}\left(b_{1}\right)=\int_{b_{1}}^{\infty} e^{-g(m)} L_{1}(x, m) d x, M_{2}\left(b_{2}\right)=\int_{b_{2}}^{\infty} L_{2}(x, m) d x$, with

$$
\begin{aligned}
& L_{1}(x, m)=\phi(x) \Phi\left(F_{1}\right)+e^{-2 m \delta} \phi\left(\frac{\sigma \sqrt{T-t} x+2 m \delta}{\sigma \sqrt{T-t}}\right) \Phi\left(F_{2}\right), \\
& L_{2}(x, m)=\phi(x) \Phi\left(G_{1}\right)+\phi\left(\frac{\sigma \sqrt{T-t} x+2 m \delta}{\sigma \sqrt{T-t}}\right) \Phi\left(G_{2}\right), \\
& F_{1}=\operatorname{sgn}(\delta) \frac{\delta\left[\sigma \sqrt{T-t} x+(T-t) \sigma^{2}\right]+m}{\sigma \sqrt{(T-t)\left(1-\delta^{2}\right)}}, \\
& F_{2}=\operatorname{sgn}(\delta) \frac{\delta\left[\sigma \sqrt{T-t} x+(T-t) \sigma^{2}+2 m \delta\right]-m}{\sigma \sqrt{(T-t)\left(1-\delta^{2}\right)}},
\end{aligned}
$$

$$
\begin{aligned}
& G_{1}=\operatorname{sgn}(\delta) \frac{\delta \sigma \sqrt{T-t} x+m}{\sigma \sqrt{(T-t)\left(1-\delta^{2}\right)}}, \quad G_{2}=\operatorname{sgn}(\delta) \frac{\delta[\sigma \sqrt{T-t} x+2 m \delta]-m}{\sigma \sqrt{(T-t)\left(1-\delta^{2}\right)}}, \\
& b_{1}=\frac{\ln K-\ln S_{t}-\left(r+0.5 \sigma^{2}\right)(T-t)+g(m)}{\sigma \sqrt{T-t}}, \\
& b_{2}=\frac{\ln K-\ln S_{t}-\left(r-0.5 \sigma^{2}\right)(T-t)+g(m)}{\sigma \sqrt{T-t}}, \\
& g(m)=\ln \Phi\left(C_{1}\right)+e^{-2 m \delta} \Phi\left(C_{2}\right), \quad m=\left|W_{2, t}\right|, \\
& C_{1}=\operatorname{sgn}(\delta) \frac{m+(T-t) \sigma^{2} \delta}{\sqrt{T-t} \sigma}, \quad C_{2}=\operatorname{sgn}(\delta) \frac{-m+(T-t) \sigma^{2} \delta}{\sqrt{T-t} \sigma}
\end{aligned}
$$

and $\operatorname{sgn}(x)$ being a standard sign function. 
Proof. A probabilistic approach is applied to derive the closed-form formula for European call option prices $C(S, t)$. Let $a=\ln K-\ln S_{t}-\left(r-0.5 \sigma^{2}\right)(T-t)+l(z)+y+z$, we then have

$$
\begin{aligned}
C(S, t) & =e^{-r(T-t)} E^{Q}\left[\left(S_{T}-K\right)^{+} \mid F_{t}\right]=e^{-r(T-t)}\left\{E^{Q}\left[S_{T} \cdot I_{\left(S_{T}>K\right)} \mid F_{t}\right]-K E^{Q}\left[I_{\left(S_{T}>K\right)} \mid F_{t}\right]\right\} \\
& =S_{t} e^{-0.5 \sigma^{2}(T-t)-l(z)-(y+z)} \int_{a}^{\infty} e^{u} f(u \mid y, z) d u-K e^{-r(T-t)} \int_{a}^{\infty} f(u \mid y, z) d u .
\end{aligned}
$$

Here, $f(u \mid y, z)$ is regarded as the conditional probability density function $f_{\sigma W_{T}+\sigma R_{T} \mid \sigma W_{t}, \sigma R_{t}}$. Clearly, to find the final pricing formula for $C(S, t)$, what we need to calculate are the two integrals in Equation (3.12) since the other terms are known by now. Therefore, $f(u \mid y, z)$ should be firstly derived as it is needed in the computation of the two integrals. Recalling Equation (3.4) in the last subsection,

$$
f_{\sigma W_{T}, \sigma R_{T} \mid \sigma W_{t}, \sigma R_{t}}=f_{\sigma W_{T} \mid \sigma W_{t}} f_{\sigma R_{T} \mid \sigma R_{t}}
$$

the conditional probability density function $f_{\sigma W_{T}+\sigma R_{T} \mid \sigma W_{t}, \sigma R_{t}}$ can be easily derived as

$$
\begin{aligned}
f(u \mid y, z) & =\int_{0}^{\infty} \frac{1}{2 \pi(T-t) \sigma^{2} \delta \sqrt{1-\delta^{2}}} e^{-\frac{(u-v-y)^{2}}{2\left(1-\delta^{2}\right) \sigma^{2}(T-t)}}\left[e^{-\frac{(v-z)^{2}}{2(T-t) \sigma^{2} \delta^{2}}}+e^{-\frac{(v+z)^{2}}{2(T-t) \sigma^{2} \delta^{2}}}\right] d v \\
& =\frac{1}{\sqrt{2 \pi(T-t)} \sigma} e^{-\frac{(u-y-z)^{2}}{2 \sigma^{2}(T-t)}} \Phi\left(a_{1}\right)+e^{-\frac{(u-y+z)^{2}}{2 \sigma^{2}(T-t)}} \Phi\left(a_{2}\right)
\end{aligned}
$$

where $a_{1}=\frac{\delta^{2}(u-y-z)+z}{\delta \sigma \sqrt{(T-t)\left(1-\delta^{2}\right)}}, \quad a_{2}=\frac{\delta^{2}(u-y+z)-z}{\delta \sigma \sqrt{(T-t)\left(1-\delta^{2}\right)}}$. As a result, the first integral in Equation (3.12) can be calculated as

$$
\begin{aligned}
\int_{a}^{+\infty} e^{u} f(u \mid y, z) d u & =\frac{1}{\sigma \sqrt{2 \pi(T-t)}} e^{y+z+0.5 \sigma^{2}(T-t)} \int_{a}^{+\infty}\left[e^{-\frac{\left[u-y-z-\sigma^{2}(T-t)\right]^{2}}{2(T-t) \sigma^{2}}} \Phi\left(a_{1}\right)\right. \\
& \left.+e^{-2 z} e^{-\frac{\left[u-y+z-\sigma^{2}(T-t)\right]^{2}}{2(T-t) \sigma^{2}}} \Phi\left(a_{2}\right)\right] d u
\end{aligned}
$$

By means of transformation of the variable $x=\frac{u-y-z-(T-t) \sigma^{2}}{\sigma \sqrt{T-t}}$, the above equation 
becomes

$$
\begin{aligned}
\int_{a}^{\infty} e^{u} f(u \mid y, z) d u & =e^{y+z+0.5 \sigma^{2}(T-t)} \int_{b_{1}}^{\infty} \phi(x) \Phi\left(\frac{\delta^{2}\left[\sigma \sqrt{T-t} x+(T-t) \sigma^{2}\right]+z}{\sigma \delta \sqrt{(T-t)\left(1-\delta^{2}\right)}}\right) \\
& +e^{-2 z} \phi\left(x+\frac{2 z}{\sigma \sqrt{T-t}}\right) \Phi\left(\frac{\delta^{2}\left[\sigma \sqrt{T-t} x+(T-t) \sigma^{2}+2 z\right]-z}{\sigma \delta \sqrt{(T-t)\left(1-\delta^{2}\right)}}\right) d x \\
& \triangleq e^{y+z+0.5 \sigma^{2}(T-t)} \Psi_{1}\left(b_{1}\right)
\end{aligned}
$$

where $b_{1}=\frac{\ln K-\ln S_{t}-\left(r+0.5 \sigma^{2}\right)(T-t)+l(z)}{\sigma \sqrt{T-t}}$. In a similar fashion, with $w=$ $\frac{u-y-z}{\sigma \sqrt{T-t}}$, we can obtain the expression of the second integral as

$$
\begin{aligned}
\int_{a}^{\infty} f(u \mid y, z) d u & =\int_{b_{2}}^{\infty} \phi(x) \Phi\left[\frac{\delta^{2} \sigma \sqrt{T-t} w+z}{\sigma \delta \sqrt{(T-t)\left(1-\delta^{2}\right)}}\right] \\
& +\phi\left(w+\frac{2 z}{\sigma \sqrt{T-t}}\right) \Phi\left[\frac{\delta^{2}(\sigma \sqrt{T-t} w+2 z)-z}{\sigma \delta \sqrt{(T-t)\left(1-\delta^{2}\right)}}\right] d w \\
& \triangleq \Psi_{2}\left(b_{2}\right)
\end{aligned}
$$

where $b_{2}=\frac{\ln K-\ln S_{t}-\left(r-0.5 \sigma^{2}\right)(T-t)+l(z)}{\sigma \sqrt{T-t}}$.

Consequently, we have $C(S, t)=S_{t} e^{-l(z)} \Psi_{1}\left(b_{1}\right)-K e^{-r(T-t)} \Psi_{2}\left(b_{2}\right)$. With $z=\delta m$, we can finally reach Equation (3.11), which has completed the proof.

The option pricing formula that we have just derived is of the same form as that of the B-S formula, except that $N_{1}\left(d_{1}\right)$ and $N_{2}\left(d_{2}\right)$ have been replaced by $M_{1}\left(b_{1}\right)$ and $M_{2}\left(b_{2}\right)$, respectively. On the other hand, the newly derived formula is different from the B-S formula with a newly added parameter $\delta$ representing skewness. When $\delta=0$, one can easily verify that it does degenerate to the B-S formula as expected. Moreover, our pricing formula depends on the initial value of $W_{2, t}$ and it is analogous to the stochastic volatility model where the option price depends on the initial value of the volatility. However, unlike stochastic volatility models, the newly proposed formula in this paper is really easy to compute since the integrals $M_{1}\left(b_{1}\right)$ and $M_{2}\left(b_{2}\right)$ can be numerically evaluated straightforwardly.

The corresponding Greeks $\Delta_{c}, \Gamma_{c}, R_{c}$, and $K_{c}$, where the subscript $c$ denotes the call 
option, can be easily worked out as those under the B-S model, and they are presented in the Appendix. In particular, one may be very interested in $\Omega_{c}$ as it is a new "Greek" as a result of the introduction of the skewness parameter $\delta$, and thus its impact on option pricing will be further studied in the next section.

\section{Numerical examples and discussions}

In this section, we firstly explore the rate of change of option prices with respect to a change of the skewness parameter, $\delta$, which is a new Greek as a result of introducing the skew feature of the underlying price observed in market data. Then, option prices calculated under the adopted model will be compared with those obtained under the B-S model.

First of all, among all Greeks, $\Omega_{c}$, which describes the influence of the skewness parameter $\delta$ on European call option prices, is the most interesting one since there is no counterpart in the B-S model. In the following study, we only vary one parameter at a time in the expression of $\Omega_{c}$ while keeping other parameters unchanged.

Depicted in Fig. 2 are the variations of $\Omega_{c}$ vs. a set of $\delta$ values varying between $-0.95 \leq \delta \leq 0.95$ with three discrete values chosen for each of the remaining 4 parameters to demonstrate the impact of these parameters on $\Omega_{c}$. It is interesting to observe from Fig. 2(a) that values of $\left|\Omega_{c}\right|$ for deeply in-the-money options are larger than those for out-of-money options when $|\delta|$ approaches 1, whereas such a sharp contrast is not so clear when $\delta$ is close to zero. In other words, this suggests that in-the-money option prices are more sensitive to the skewness of the probability density function of the underlying asset when $|\delta|$ is close to 1 . On the other hand, the variation of $\left|\Omega_{c}\right|$ taking the risk-free interest rate as a parameter seems to display something quite different. As shown in Fig. 2(b), changes of $\delta$ have almost the same effect on option prices when the risk-free interest rate $r$ varies below 0.1 . Furthermore, the variations of $\left|\Omega_{c}\right|$ taking the volatility and time to expiration as a parameter are shown in Fig. 2(c) and 2(d), respectively. Obviously, the larger $\sigma$ or $T-t$ is, the more impacts the changing $\delta$ will have on the option price in the 


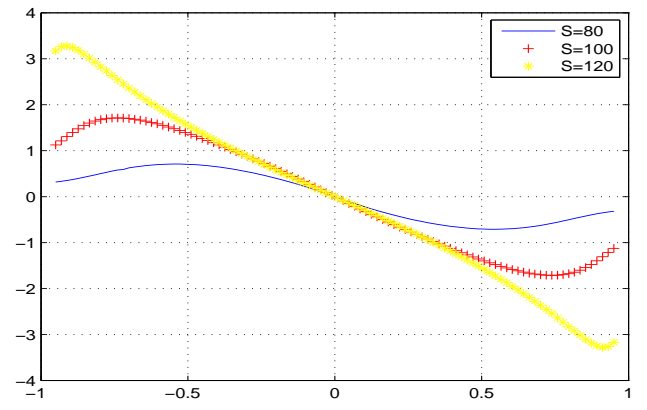

(a) Changes of $\Omega_{c}$ with three different values of $S$.

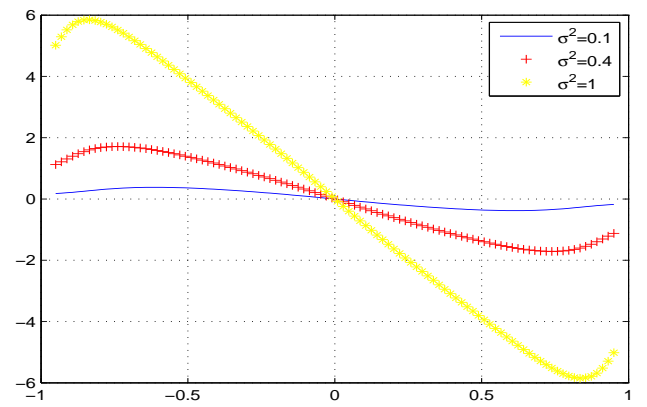

(c) Changes of $\Omega_{c}$ with three different values of $\sigma$. (d) Changes of $\Omega_{c}$ with three different values of $T-$

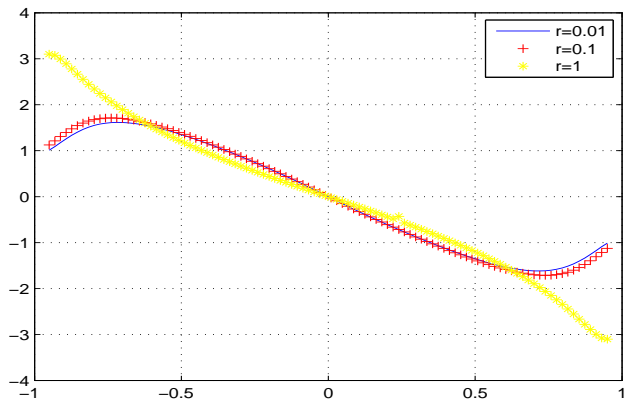

(b) Changes of $\Omega_{c}$ with three different values of $r$.

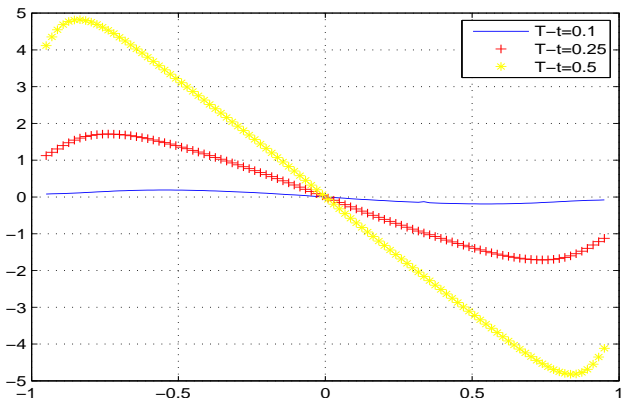

Figure 2: Changes of $\Omega_{c}$ according to $\delta$

whole region of $-0.95 \leq \delta \leq 0.95$.

One of the most important common features, which is shown in all the four figures in Fig. 2, is that $\Omega_{c}$ is negative when $\delta$ is positive and vice versa, which means that option prices are a monotonically increasing function of $\delta$ for negative $\delta$ values whereas they are a a monotonically decreasing function for positive $\delta$ values. From this, we can conclude that the maximum option price is reached when $\delta=0$, which is exactly the B-S price.

What is shown in Fig. 3 is the comparison of option prices obtained from the newly derived formula and B-S prices. It can be easily observed from Fig. 3(a) and 3(b) that $S$ and $r$ have a similar influence on option prices calculated with our formula and those obtained through the B-S formula, while in Fig. 3(c) and 3(d) the difference between our prices and B-S prices is enlarged when the value of volatility $\sigma$ or time to expiration $T-t$ increases. Collectively, all the figures in Fig. 3 confirm that the trend of option prices 
calculated with our formula is indeed the same as what we have shown in the Appendix. In particular, all our prices are lower than B-S prices, which can be partly explained by the decrease in the variance of stock log-price when the absolute value of $\delta$ increases.

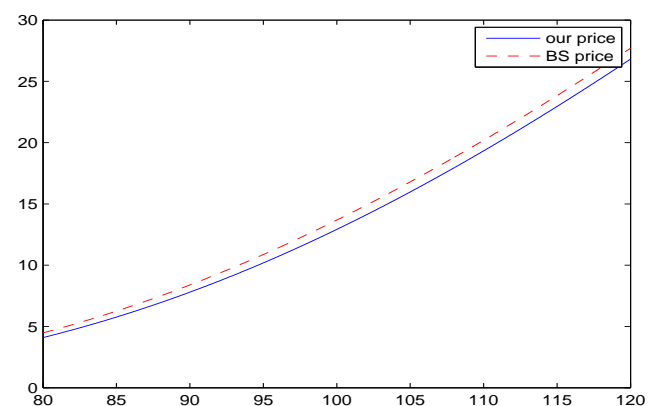

(a) Our price vs B-S price when $S \in(80,120)$.

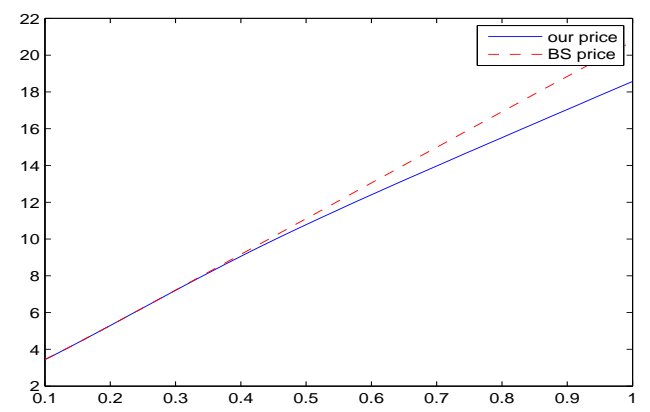

(c) Our price vs B-S price when $\sigma \in(0.1,1)$.

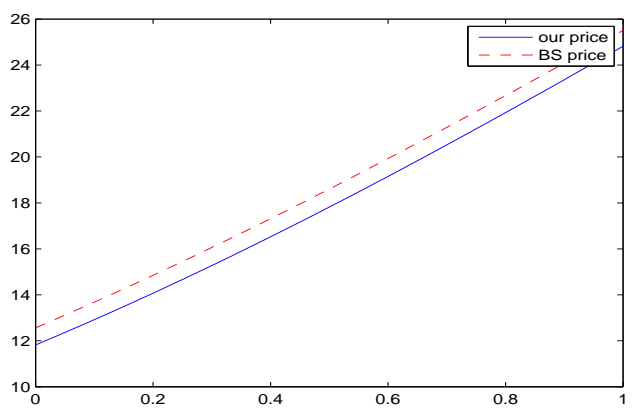

(b) Our price vs B-S price when $r \in(0,1)$.

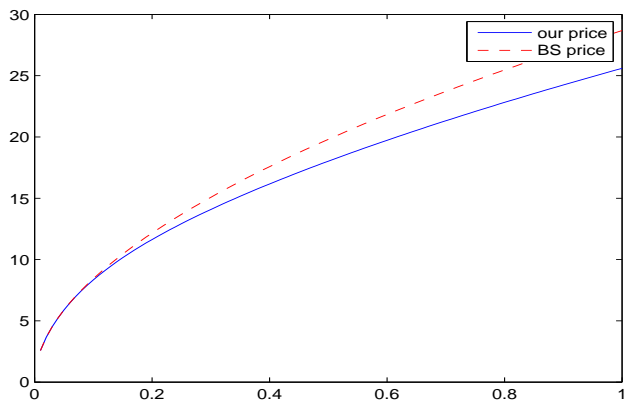

(d) Our price vs B-S price when $T-t \in(0.01,1)$.

Figure 3: Our price vs B-S price for different ranges of different parameters

\section{Conclusion}

In this paper, a new European call option pricing formula is derived when the dynamics of the underlying are modeled by a special kind of skew Brownian motions, which is introduced to capture the non-normal property of the distribution of log-returns. Upon identifying two errors in a key reference in this area, we have introduced a new equivalent martingale measure named "endogenous risk neutral measure", based on which the analytical tractability of the problem is preserved and a new closed-form pricing formula is obtained. Using the newly derived formula, the quantitative impacts of the skewness parameter $\delta$ on option prices are discussed through numerical experiments. 


\section{References}

[1] A. Azzalini. A class of distributions which includes the normal ones. Scandinavian Journal of Statistics, pages 171-178, 1985.

[2] F. Black and M. Scholes. The pricing of options and corporate liabilities. The Journal of Political Economy, pages 637-654, 1973.

[3] P. Carr, H. Geman, D. B. Madan, and M. Yor. The fine structure of asset returns: An empirical investigation*. The Journal of Business, 75(2):305-333, 2002.

[4] T. Corns and S. Satchell. Skew brownian motion and pricing european options. The European Journal of Finance, 13(6):523-544, 2007.

[5] M. Eling, S. Farinelli, D. Rossello, and L. Tibiletti. Skewness in hedge funds returns: classical skewness coefficients vs azzalini's skewness parameter. International Journal of Managerial Finance, 6(4):290-304, 2010.

[6] M. Frittelli. The minimal entropy martingale measure and the valuation problem in incomplete markets. Mathematical Finance, 10(1):39-52, 2000.

[7] A. Gairat and V. Shcherbakov. Density of skew brownian motion and its functionals with application in finance. Mathematical Finance, 2016.

[8] K. Itô and H. McKean Jr. Diffusion processes and their sample paths, 1965.

[9] D. Kim and S. J. Kon. Structural change and time dependence in models of stock returns. Journal of Empirical Finance, 6(3):283-308, 1999.

[10] S. G. Kou. A jump-diffusion model for option pricing. Management Science, 48(8):1086-1101, 2002.

[11] D. B. Madan, P. P. Carr, and E. C. Chang. The variance gamma process and option pricing. European Finance Review, 2(1):79-105, 1998.

[12] R. C. Merton. Option pricing when underlying stock returns are discontinuous. Journal of Financial Economics, 3(1):125-144, 1976.

[13] W. Nilsen and H. Sayit. No arbitrage in markets with bounces and sinks. International Review of Applied Financial Issues \& Economics, 3(4):696-699, 2011.

[14] A. Peiro. Skewness in financial returns. Journal of Banking $\mathcal{E}$ Finance, 23(6):847-862, 1999.

[15] T. H. Rydberg. The normal inverse gaussian lévy process: simulation and approximation. Communications in Statistics. Stochastic Models, 13(4):887-910, 1997.

[16] M. Schweizer. On the minimal martingale measure and the möllmer-schweizer decomposition. Stochastic Analysis and Applications, 13(5):573-599, 1995. 


\section{Appendix}

The Greeks of European call option prices under the adopted model are

$$
\begin{aligned}
\Delta_{c} & =\frac{\partial C}{\partial S}=M_{1}\left(b_{1}\right)>0 \\
\Gamma_{c} & =\frac{\partial^{2} C}{\partial^{2} S}=\frac{e^{-g(m)} L_{1}\left(b_{1}, m\right)}{S \sigma \sqrt{T-t}}>0 \\
R_{c} & =\frac{\partial C}{\partial r}=K e^{-r(T-t)} M_{2}\left(b_{2}\right)>0 \\
K_{c} & =\frac{\partial C}{\partial K}=-e^{-r(T-t)} M_{2}\left(b_{2}\right)<0 \\
\Omega_{c} & =\frac{\partial C}{\partial \delta}=S I_{1}-K e^{-r(T-t)} I_{2}-S I_{3},
\end{aligned}
$$

where $I_{i}, i \in 1,2,3$ are defined as follows:

$$
\begin{aligned}
I_{1} & =e^{-g(m)} \int_{b_{1}}^{\infty} \phi(x) \phi\left(F_{1}\right) \operatorname{sgn}(\delta) \frac{x+\sqrt{T-t} \sigma+\frac{m \delta}{\sigma \sqrt{T-t}}}{\left(1-\delta^{2}\right)^{1.5}} \\
& +\phi\left(\frac{\sigma \sqrt{T-t} x+2 m \delta}{\sigma \sqrt{T-t}}\right) \Phi\left(F_{2}\right) e^{-2 m \delta}\left[-2 m-\frac{-2 m(\sigma \sqrt{T-t} x+2 m \delta)}{\sigma^{2}(T-t)}\right] \\
& +\phi\left(\frac{\sigma \sqrt{T-t} x+2 m \delta}{\sigma \sqrt{T-t}}\right) \phi\left(F_{2}\right) e^{-2 m \delta}\left[\operatorname{sgn}(\delta) \frac{x+\sigma \sqrt{T-t}+\frac{\left(3 \delta-2 \delta^{3}\right) m}{\sigma \sqrt{T-t}}}{\left(1-\delta^{2}\right)^{1.5}}\right] d x, \\
I_{2}= & \int_{b_{1}}^{\infty} \phi(x) \phi\left(G_{1}\right) \operatorname{sgn}(\delta) \frac{x+\frac{m \delta}{\sigma \sqrt{T-t}}}{\left(1-\delta^{2}\right)^{1.5}} \\
& +\phi\left(\frac{\sigma \sqrt{T-t} x+2 m \delta}{\sigma \sqrt{T-t}}\right) \Phi\left(G_{2}\right)\left[-\frac{2 m(\sigma \sqrt{T-t} x+2 m \delta)}{\sigma^{2}(T-t)}\right] \\
& +\phi\left(\frac{\sigma \sqrt{T-t} x+2 m \delta}{\sigma \sqrt{T-t}}\right) \phi\left(G_{2}\right)\left[\operatorname{sgn}(\delta) \frac{x+\frac{\left(3 \delta-2 \delta^{3}\right) m}{\sigma \sqrt{T-t}}}{\left(1-\delta^{2}\right)^{1.5}}\right] d x, \\
I_{3} & =\int_{b_{1}}^{\infty} \frac{L_{1}(x, m)}{e^{2 g(m)}\left[\sigma \sqrt{T-t} \operatorname{sgn}(\delta)\left(\phi\left(C_{1}\right)+e^{-2 m \delta} \phi\left(C_{2}\right)\right)-2 m e^{-2 m \delta} \Phi\left(C_{2}\right)\right] d x .}
\end{aligned}
$$

